\title{
Cidades Saudáveis e Cidades Inteligentes: uma abordagem comparativa
}

\author{
Healthy Cities And Smart Cities: A comparative aproach
}

\author{
Lidiane Aparecida Alves ${ }^{1}$
}

\begin{abstract}
Resumo
O presente artigo tem como objetivo apresentar, comparar e analisar as noções de Cidades Saudáveis e Cidades Inteligentes. Para seu alcance, a metodologia utilizada consistiu em uma revisão da literatura, que poderá contribuir com as discussões científicas e ampliação da compreensão sobre as Cidades Saudáveis e/ou Inteligentes. Entre estas noções, há diferenças e pontos em comuns, conforme foi evidenciado neste artigo. Guardadas as particularidades, em ambas o que se busca é a sustentabilidade, a qualidade de vida, a saúde e o bem-estar a partir de intervenções no ambiente e valorização do capital social, em uma sociedade urbana e globalizada. Reitera-se que a materialização desta busca depende do esforço de vários setores, instituições e de políticas intersetoriais.
\end{abstract}

Palavras-chave: Cidades. Conceitos. Sustentabilidade. Urbanização. Globalização.

\begin{abstract}
This article aims to present, compare and analyze the notions of Healthy Cities and Smart Cities. To reach it, the methodology used consisted of a review of the literature, which could contribute to the scientific discussions and increase understanding about Healthy and/or Smart Cities. Among these notions, there are differences and points in common, as evidenced in this article. Keeping in mind the particularities, in both what is sought is sustainability, quality of life, health and well-being from interventions in the environment and valorization of social capital in an urban and globalized society. It is reiterated that the materialization of this search depends on the efforts of various sectors, institutions and intersectoral policies.
\end{abstract}

Keywords: Cities. Concepts. Sustainability. Urbanization. Globalization.

\section{Introdução}

Nos últimos anos do século XX e início do século XXI foram várias as transformações no âmbito da sociedade, designada urbano-industrial. O

\footnotetext{
${ }^{1}$ Universidade de Federal de Uberlândia, Uberlândia, Minas Gerais, Brasil. lidianeaa@yahoo.com.br Artigo recebido em: 25/04/2019. Aceito para publicação em: 28/08/2019.
} 
rápido crescimento da população urbana, por vezes, materializado desacompanhado do provimento dos recursos necessários para o atendimento das necessidades básicas, associado às mudanças socioeconômicas, ambientais e tecnológicas, em escala planetária, haja vista o processo de globalização, conferem à sociedade atual uma gama de desafios. No entanto, há também oportunidades para mudanças positivas.

A superação dos desafios e a busca por infraestruturas urbanas melhores, mais eficientes e com menores custos, requer considerar a complexidade inerente às cidades no contexto do capitalismo globalizado e explorar as novas oportunidades como as tecnologias, inclusive para o aumento da participação social e da cooperação horizontal tendo como meta o alcance do desenvolvimento socioespacial e de condições a ele relacionadas como a melhoria do nível de saúde, da qualidade de vida e da sustentabilidade na/da cidade.

As cidades são locais de concentração de capital físico e humano, condição que pode ser potencializada pela utilização da inovação e de tecnologias para atrair moradores, visitantes e investimentos, transformando-as em polos de competitividade global. Governos, moradores, empresas/indústrias e universidades, estão interessados na construção de cidades que combinem a competitividade geopolítica e a sustentabilidade. (ANTHOPOULOS, 2014; MOSANNENZADEH; VETTORATO, 2014).

Neste contexto, sob diferentes designações e formas de abordagens, ganham destaque movimentos em torno de cidades designadas por conceitos como saudáveis, inteligentes, entre outros. Mesmo considerando a diversidade de enfoques, especialmente em relação às Cidades Inteligentes, à primeira vista, as noções de Cidades Saudáveis e Inteligentes estariam muito próximas, pois em ambas o foco são os ganhos em termos de qualidade socioespacial a partir de soluções nos serviços e infraestrutura urbanos.

De fato há similaridades como esta e outras, a exemplo do protagonismo que o cidadão deve assumir e da natureza contínua das 
melhorias, ainda que no âmbito da noção de Cidades Saudáveis isto esteja mais explícito. Outro ponto em que as noções se assemelham é ao considerarem que a condição de saudável ou inteligente está sempre passível a sucessivas evoluções consonante com os anseios e atuação dos cidadãos. Entretanto, observa-se a existência de pontos distintos, sobretudo ao considerar as características almejadas para os serviços e infraestrutura urbana e o foco principal de cada noção. Observa-se explicita e/ou implicitamente que, enquanto nas Cidades Saudáveis busca-se a oferta de uma infraestrutura urbana acessível que atenda as demandas dos moradores, nas Cidades Inteligentes almeja-se que a infraestrutura e serviços urbanos sejam conectados em conformidade com as demandas de uma sociedade baseada nas tecnologias.

O presente artigo tem como objetivo apresentar, comparar e analisar as noções de Cidades Saudáveis e Cidades Inteligentes, tendo em vista o relevo, tanto no âmbito teórico como prático, que tais noções têm assumido hodiernamente. De tal modo, para o alcance do objetivo proposto, a metodologia utilizada na pesquisa consistiu em uma revisão da literatura, onde se considerou estudos acadêmicos e documentos como relatórios sobre projetos e exemplos de iniciativas de implementação concebidos por municípios, instituições governamentais e grandes empresas. Este estudo justifica-se pela contribuição para com as discussões sobre as noções de Cidades Saudáveis e Inteligentes, considerando a necessidade da abordagem científica para compreender tais conceitos. Além disso, como as inciativas de Cidades Saudáveis ou Inteligentes têm sido específicas para cada local e, de modo geral, contemplando apenas parte da cidade, este estudo buscou apresentar uma visão geral da aplicabilidade dos conceitos. 


\section{A noção de Cidades Saudáveis}

O conceito de Cidade Saudável exprime um Movimento da Organização Mundial da Saúde (OMS) originado em 1978, na América do Norte (Toronto/Canadá) e difundido em um primeiro momento na Europa e posteriormente nos Estados Unidos da América (EUA), com abrangência global nos dias atuais. Dentre os seus pressupostos está colocar a saúde local no centro da agenda política, econômica e social, melhorando-a por meio de modificações dos ambientes físico, social e econômico. A definição fundamental de Cidades Saudáveis, elaborada por Hancock e Duhl (1986) é,

[...] aquela que está continuamente a criar e melhorar os ambientes físicos e sociais e fortalecer os recursos da comunidade que permitem às pessoas apoiarem-se mutuamente na realização de todas as funções da vida e alcançar o seu potencial máximo. (HANCOCK; DUHL, 1986, apud HANCOCK, 1993, p.7. Tradução Nossa).

O conceito foi apoiado pela OMS e embasa-se nos princípios de Atenção Primária à Saúde, Promoção da Saúde, Saúde para Todos e saúde mais do que a ausência de doença, enfatizando a interação entre as características físicas, dimensões mental e social, incorporando recentemente as "estratégias e metas de saúde para o século XXI" da Agenda 21 Local, e as recomendações da Conferência das Nações Unidas sobre Assentamentos Humanos. A potencialidade das ações no nível local para resolver problemas de saúde urbana em países industrializados e em desenvolvimento é enfatizada no âmbito da noção de Cidades Saudáveis (WHO, 1997; MYFANWY, 2010).

Em função de diferenças em termos de condições sociais, econômicas e naturais o conceito "saudável" pode assumir diferentes acepções, o que implica em enfoques e áreas prioritárias diversas. Neste sentido, como exemplifica Righetti (2004) a limpeza das cidades e aumento de coleta de lixo reciclável foi enfoque na Costa Rica; o consumo de alimentos saudáveis 
foi priorizado em Cuba e as campanhas pela redução do consumo de álcool e contra o porte de armas, visando diminuir os altos índices de criminalidade na Colômbia, sobretudo em Bogotá.

Como requisitos gerais para o estabelecimento de uma Cidade Saudável, Duhl (1986) delineia que a cidade ofereça respostas adequadas e eficazes para as necessidades de desenvolvimento das organizações e do povo; que a cidade tenha a capacidade de modificar-se para atender às exigências emergentes; que capacite seus habitantes a usá-la em favor do bem-estar, o que remete a educação dos seus cidadãos.

Deste modo, um compromisso com a saúde, um processo e estrutura para alcançá-lo, bem como a melhoria contínua dos ambientes e dos recursos físicos e sociais, definem uma Cidade Saudável. E, não o estado de saúde atual ou mesmo fato de ter alcançado um estado de saúde particular. Tal como ocorre para o alcance da sustentabilidade, o relevante é a adoção de ações que levem ao objetivo pretendido. Neste sentido, nos vários estudos é consensual a relevância de um pacto social de compromisso com a promoção da saúde, visando à melhoria da qualidade de vida da população, em que a chave é a mudança de atitude quanto aos modos de promover a saúde, mudando as políticas, legislações e serviços providos pelo município.

Conforme destaca a WHO (2013b), o Movimento Cidades Saudáveis promove políticas e planejamento abrangentes e sistemáticas para a saúde e enfatiza: (1) a necessidade de abordar a desigualdade na saúde e pobreza urbana; (2) as necessidades dos grupos vulneráveis; (3) governança participativa; (4) planejamento e o design urbano saudável; e (5) os determinantes sociais, econômicos e ambientais da saúde.

A concepção de saúde como processo, que a partir do controle de determinados fatores pode ser melhorada, reconhecido na Carta de Ottawa, implica na necessidade de novas habilidades, processos, estilos e estruturas para o processo de governança, a qual é 
[...] constituída pelos seguintes elementos de ação: i) forte liderança e apoio de prefeitos; ii) apoio de todos os partidos em conselhos municipais; iii) acordos de parceria com setores estatutário e não estatutário; iv) uma variedade de estruturas e processos para suportar inter cooperação e envolvimento dos cidadãos; [...] v) o planejamento estratégico para direcionar-definir; vi) e rede formal e informal. (WHO, 2013, p.58).

Assim, segundo a WHO, a equidade, a governança participativa e a solidariedade, a colaboração intersetorial e de ação são essenciais às Cidades Saudáveis. Um projeto para a Cidades Saudávelsegundo Hall; Davies e Sherriff (2010) rejeita a abordagem top-down (a solução de engenharia física e social) em favor de uma nova perspectiva sobre os problemas de saúde urbanos e foco em estratégias baseadas na comunidade bottom-up para combater questões saúde pública. Deste modo, para a consecução da Cidade Saudável é demandado: compromisso político explícito, liderança, mudança institucional além de parcerias intersetoriais, o que de acordo com a WHO (2014, s/p) demanda de "envolvimento da população local no processo de tomada de decisão, um compromisso político e desenvolvimento organizacional e comunitário". A WHO (2014) destaca ainda que o processo é tão importante quanto os resultados que serão visíveis em longo prazo.

Portanto, as Cidades Saudáveis da rede da WHO são aquelas que estão comprometidas com a saúde e o desenvolvimento sustentável, com foco nas áreas da saúde, economia e desenvolvimento urbano, através de um processo "de compromisso político, mudança institucional, capacitação, planejamento baseado em parcerias e projetos inovadores", que envolve os governos locais em um esforço contínuo para a melhoria. Ao considerar a complexidade e dinamicidade da cidade, que está em constante transformação, o projeto Cidades Saudáveis considera o estado atual da cidade, porém com uma visão de que esta pode se tornar uma Cidade Saudável, com a melhoria os seus ambientes e expansão dos seus recursos para as pessoas apoiarem-se mutuamente na realização de seu potencial mais elevado (WHO,1997). 
A Organização Panamericana de Saúde (OPAS) define um município saudável como aquele em que as autoridades políticas e civis, as instituições e organizações públicas e privadas, os proprietários, empresários, trabalhadores e a sociedade dedicam constantes esforços para melhorar as condições de vida, trabalho e cultura da população; estabelecem uma relação harmoniosa com o meio ambiente físico e natural e expandem os recursos comunitários para melhorar a convivência, desenvolver a solidariedade, a cogestão e a democracia (OPAS, 1996).

Mendes (1996) foi um dos primeiros autores brasileiros a abordar a questão das Cidades Saudáveis, considerando-as como projeto estruturante do campo da saúde. Além disso, a disseminação do ideário de Cidades Saudáveis iniciou-se nas escolas de saúde pública na década de 1980, fortalecendo no setor de saúde com a criação do Sistema Único de Saúde (SUS) nos anos 1990, que têm seus pressupostos convergentes com os das Cidades Saudáveis.

\section{A noção de Cidades Inteligentes}

O conceito de Smart Cities, Cidades Inteligentes em português, não é uma novidade do século XXI, mas tem raízes no século XX. De acordo com alguns autores, sua origem está relacionada ao aumento do emprego de métodos científicos e aplicação de análise computacional no planejamento urbano a partir do pós-guerra, nos anos de 1950 (SHELTON; ZOOK e WIIG, 2015). Para outros estudiosos, sua origem deriva da busca por um novo planejamento, tendo surgido na literatura mais tarde, nos anos de 1990. No século XXI, o conceito passou a ser utilizado pelo setor empresarial através do aproveitamento da internet e da tecnologia Web 2.0 para a melhoria das infraestruturas das cidades (DAMERI, 2013; ANTHOPOULOS, 2015).

O fato é que, nos últimos anos, tal conceito tem ganhado força em todas as regiões do mundo. Além, disso é bastante difuso e empregado a 
partir de várias perspectivas de acordo com quem o usa, com as particularidades de cada local e com vistas a enfrentar os múltiplos desafios urbanos. Nas palavras de Anthopoulos (2015, p. 140)

[...] Cidade Inteligente não descreve uma cidade com atributos particulares, mas é usada para descrever diferentes casos em espaços urbanos: portais que virtualizam cidades ou guias de cidades, bases de conhecimento que atendem às necessidades locais, aglomerações com Tecnologias de Informação e Comunicação (TIC), infraestrutura que atrai a deslocalização de negócios, infraestruturas de TIC metropolitanas que fornecem serviços eletrônicos para os cidadãos, ambientes ubíquos e, recentemente, infraestrutura de TIC para uso ecológico.

A International Business Machines (IBM, s/d), que implantou tecnologias no Rio de Janeiro (Brasil), Zhenjiang (China), Dubuque (Dubai), Nova York (EUA), Dublin (Irlanda) e Nairóbi (Quênia) etc. afirma que uma Cidade Inteligente é aquela que aplica tecnologias de informação para o planejamento, concepção, construção e operação de infraestrutura da cidade. Conforme a Comissão Europeia (2015) uma Cidade Inteligente pressupõe construir soluções eficientes e úteis nos domínios dos serviços tradicionais de uma cidade, como os da educação, da saúde, do ambiente, da gestão de recursos (água e energia) e dos sistemas de mobilidade, do tratamento de resíduos, da segurança e acessibilidade dos espaços públicos e da administração municipal. Desta forma, de acordo com Nam e Pardo (2011), ainda que muitas vezes a inovação tecnológica seja mais enfocada do que a inovação em gestão e política, a ideia de Cidade Inteligente sugere uma interação contextualizada de inovações tecnológicas, na gestão e na política.

A Rede Brasileira de Cidades Inteligentes e Humanas associa o conceito de humanas ao de inteligente e define as cidades com estas qualidades como:

[...] aquelas que se dotam de uma infraestrutura tecnológica interoperável, necessária para conectar todos os hardwares, softwares e aplicações existentes ou que venham a existir, de uma 
maneira que se transformem em uma plataforma que funcione como um nó que conecte todas as demais plataformas, permitindo à cidade que integre todos os dados e informações gerados, para ter um sistema de informações gerenciais aberto e transparente, de uma maneira que a tecnologia sirva de apoio à melhora da qualidade de vida das pessoas, sempre com sua participação em um processo co-criativo com o poder público. (REDE BRASILEIRA DE CIDADES INTELIGENTES E HUMANAS, 2017, s/p.).

Mosannenzadeh e Vettorato (2014) ao realizarem uma revisão de literatura constataram que o conceito de Cidade Inteligente foi desenvolvido em três áreas principais: (1) Acadêmica, (2) Industrial e (3) Governamental, sendo que em cada uma predomina diferentes pontos de vista e interesses, bem como entendimentos da palavra "inteligente".

Parte significativa das visões do que consiste uma Cidade Inteligente converge para o sentido de que em tais cidades as infraestruturas e serviços são baseados em inovação, não se restringindo às Tecnologias da Informação e Comunicação TIC's (DAMERI 2013; ANTHOPOULOS, 2015), ainda que estas sejam essenciais (LEE et al, 2013.; ODENDAAL, 2003 apud MOSANNENZADEH; VETTORATO, 2014) e, de modo geral, utilizadas para permitir a produção de "big data" passível de ser utilizado, inclusive em tempo real, em prol do aumento da eficiência da cidade.

Há que considerar que, além do entendimento de Cidades Inteligentes a partir das inovações e da implantação de vários serviços eletrônicos em áreas urbanas (ANTHOPOULOS; VAKALI, 2012), o conceito pode ser usado para referir-se à e-governança (VAN DER MEER; VAN WINDEN, 2003); à aprendizagem ou conhecimento (CAMPBELL, 2012; MCFARLANE, 2011); às Cidades Criativas (FLÓRIDA, 2010); ao compartilhamento de dados abertos (BATES, 2013). Ao considerar a proeminente valorização das iniciativas que consideram a sustentabilidade a noção aparece vinculada ao consumo inteligente de energia, transporte e outros tipos de gestão de ativos rígidos (NEIROTTI et al, 2014.); às saídas de baixo carbono (BULKELEY et al, 2010) às discussões sobre Eco ou Cidades Verdes (BEATLEY; NEWMAN, 2008; JOSS et al, 2013), ao consumo de energia e pegada ambiental 
(COHEN, 2012) e ao "smartness footprint" de uma cidade que pode ser medido por um conjunto de índices (GIFFINGER et al., 2007). Há ainda autores, como Moyser (2013) e Comstock (2012), que enfatizam o governo e os serviços urbanos, por considerar que estes são os níveis mais básicos de uma cidade.(ANTHOPOULOS; FITSILIS, 2014; HOLLANDS 2014).

Outra questão que converge em várias abordagens acerca das Cidades Inteligentes é o papel fundamental do cidadão, seja em relação à participação democrática e controle social no processo de planejamento e gestão urbano e/ou na contribuição com informações e avaliações ou como usuário final dos serviços, especialmente os providos de TIC's. Os cidadãos devem ser protagonistas na apropriação das inovações tecnológicas para proporcionar melhor qualidade de vida, em termos da resolução de problemas objetivos e satisfação das necessidades objetivas e subjetivas. Neste sentido, autores como Hollands (2014) corrobora com Hoornweg (2011), Harvey (2012) e Anttiroiko (2013) no sentido de que o centro das inovações urbanas deve ser o cidadão, haja vista que os principais problemas urbanos têm bases sociológicas e não tecnológicas. Segundo Hollands (2014) deve-se ampliar o espaço para as pessoas liderarem iniciativas inteligentes e debaterem democraticamente seus direitos e as soluções para os problemas urbanos. Assim, uma Cidade Inteligente seria acolhedora, inclusiva e aberta.

Dameri (2013) considera como os componentes de uma Cidade Inteligente o meio físico e social, as tecnologias, não só as TICs, mas de vários tipos como aquelas voltadas para a produção de eletricidade e aquecimento. Para a referida autora, a transformação da cidade e, por conseguinte constituição de uma Cidade Inteligente abrange mudanças nas características das edificações e dos transportes que devem ser eficientes, assim como nos valores e iniciativas dos cidadãos e dos governos.

Apesar de pequenas variações, as medidas inerentes à uma Cidade Inteligente, ou seja, o uso dos recursos informacionais nos sistemas de 
transporte, energia, saneamento etc. em última análise, convergem com os ideais de inclusão, conectividade, encurtamento das distâncias e compartilhamento, assim como demandam de esforço e planejamento, de modo integrado, para serem implementadas. Neste sentido, ao considerar a forma de proposição e construção de uma Cidade Inteligente, identifica-se tanto abordagens de baixo para cima, a partir dos cidadãos, como de cima para baixo, a partir dos planejadores e gestores, ou integradas.

Dameri (2013) destaca uma abordagem de cima para baixo a partir de uma visão estratégica de uma Cidade Inteligente desenvolvida aplicando as regras do governo e políticas, para alcançar determinados objetivos em torno da melhoria dos problemas urbanos. E também uma abordagem de baixo para cima, que na visão da autora tem como base a aplicação da tecnologia aos problemas urbanos, tendo em conta que o acesso potencial do cidadão à cidade pode ser ampliado a partir da qualificação tecnológica das atividades urbanas, bem como que os cidadãos podem contribuir com dados que sirvam para a solução colaborativa de problemas comuns.

Hollands (2014) evidencia a concentração de Cidades Inteligentes, desenvolvidas por corporações de TIC na Ásia, especialmente no Japão e na Coreia do Sul. Dentre as quais, pode-se citar o projeto da Cisco, em parceria com a Gale International, empresa de imóveis dos EUA, na criação de New Songdo City, na Coreia do Sul, e o projeto da Panasonic na criação de Fujisawa Sustainable Smart Town (Fujisawa SST) no Japão.

No Brasil a aparição da noção de Cidades Inteligentes é mais recente e as iniciativas mais restritas, sendo os marcos a criação da Rede Brasileira de Cidades Inteligentes e Humanas em 2013, da comissão Interministerial para tratar do assunto, coordenada pela ABDI (Agência Brasileira de Desenvolvimento Industrial) em 2015 e da Frente Parlamentar Mista em “Apoio às Cidades Inteligentes e Humanas" em 2016.

Destaca-se no Brasil a emergência de projetos e iniciativas-piloto de Cidades Inteligentes, como os de Laguna-CE; de Palhoça, SC; e de 
Uberlândia-MG, onde são propostas medidas, como o uso de energia solar e das redes inteligentes, a desburocratização para facilitar o empreendedorismo, os aplicativos voltados para a mobilidade urbana, entre outros.

Localizada no distrito de Croatá, município de São Gonçalo do Amarante, no estado do Ceará destaca-se a Smart City Laguna, pensada para ser uma Cidade Inteligente social, haja vista que pauta nos princípios de inclusão e acessibilidade, e por conseguinte na busca pela sustentabilidade e pela qualidade de vida dos seus habitantes. No projeto de Laguna-CE é previsto o uso misto do solo, com áreas residenciais, de atividades de comércio e serviços, de lazer próximas, de modo a reduzir a necessidade de grandes deslocamentos diários. Além disso, existem instalações de sensores em quase tudo, desde postes de luz e lixeiras a serviços públicos e um aplicativo que unificará toda a tecnologia utilizada na cidade e acessível aos cidadãos por meio de smartphones. (AUTOMATIC HOUSE, 2019).

No caso do município de Palhoça, na grande Florianópolis, Santa Catarina, a partir do projeto chamado "Masterplan Pedra Branca", foi concebido o bairro "Pedra Branca" com quarteirões de uso misto, a ser desenvolvido até o ano de 2020, A fundamentação para o projeto urbanístico é a busca pela qualidade de vida dos moradores, que devem morar, trabalhar e se divertir sempre em locais próximos, deslocando-se de bicicleta ou à pé (AUTOMATIC HOUSE, 2019).

Cabe destacar também o pioneirismo da cidade de Uberlândia-MG, que a partir da iniciativa de uma empresa privada de capital local, a Algar Telecom, em parceria com outras empresas e instituições como: Nokia, C.E.S.A.R., PromonLogicalis e a Universidade Federal de Uberlândia, terá o bairro Granja Marileusa, que era uma fazenda da Algar, como protótipo de uma Cidade Inteligente. São propostas para este bairro inovações no âmbito do transporte coletivo (easybus, que permitirá ao passageiro conhecer a 
lotação e fluxo de passageiros); na coleta de resíduos sólidos (lixeiras inteligentes, que medirão o volume de lixo descartado e o espaço disponível, bem como permitirão ao usuário acumular pontos a partir da destinação corretas dos resíduos) e no escoamento pluvial (bueiros inteligentes, que permitirão conhecer o volume nele retido), por fim, considerando os locais onde o acesso à internet é restrito a ideia é usar balões de conectividade como solução (LIMA, 2017).

Além disso, o bairro Granja Marileusa, aprovado pela Prefeitura Municipal de Uberlândia no final do ano de 2012, foi planejado segundo a concepção urbanistíca de uso misto do solo. Portanto, com a instalação de diversas tipologias de empreendimentos, como os espaços residenciais, os espaços de comércio e de serviços, os espaços acadêmicos, os espaços de convivência, etc. A adoção de tal concepção urbanística tem como objetivos a otimização do tempo gasto em deslocamentos cotidianos, poupar os recursos naturais a partir da adoção de atitudes ecologicamente sustentáveis e resgatar o convívio entre os moradores nos espaços públicos.

Os projetos de Cidades Inteligentes ainda são pontuais, em partes da cidade. Contudo, tendo em vista os possíveis resultados positivos destes projetos, posto que se fundamentam na sustentabilidade, considerando os aspectos humanos, econômicos e tecnológicos, espera-se ampliem tanto em número de cidades como no âmbito do espaço urbano. Além disso, as Cidades Inteligentes e Saudáveis devem orientar o processo de urbanização no século XXI, que deve pautar na participação dos agentes produtores do espaço e no planejamento voltado para a mobilidade, a saúde, sustentabilidade, etc.

Portanto, ao considerar os projetos e iniciativas-piloto de Cidades Inteligentes, observa-se que eles são tendência para desenvolvimento de centros urbanos no século XXI, sendo que, de modo geral convergem com as mudanças estruturais dos espaços intraurbanos, ou seja, objetivam a criação de novas centralidades urbanas e, por conseguinte reforçam a 
reestruturação urbana a partir do rompimento com o monocentrismo em detrimento à complexificação da centralidade urbana, avigorando, conforme termo utilizado por Lefebvre (1999) a poli(multi)centralidade das cidades.

\section{Cidades Saudáveis e Cidades Inteligentes: Similaridades e diferenças}

A qualidade de vida, o bem-estar e a sustentabilidade dependem das características das cidades, que por meio de soluções no planejamento podem inovar, aproveitar suas capacidades e promover o alcance e o entrelaçamento de tais condições. Afinal, segundo Myfanwy (2010) as cidades possuem funcionamento sistêmico, em constante modificação para realizar os ajustes exigidos e modificar-se para satisfazer as exigências sempre emergentes de seus habitantes.

Ao considerar tais demandas emergentes nas cidades no contexto atual, segundo Dameri (2013), há vários conceitos usados para se referir ao uso de inovações para melhorar as cidades, como Cidades Inteligentes, Cidades Sustentáveis, Cidades Saudáveis etc. Apesar de algumas diferenças, nem sempre eles se contradizem uns com os outros, pois compartilham alguns aspectos, como a participação social e busca pela qualidade de vida, e são parcialmente sobrepostos. Por exemplo, o uso de tecnologia, Cidade Inteligente, permite a eficiência no uso de energia e redução de $\mathrm{CO} 2$, Cidade Sustentável, que por sua vez é condição para um ambiente físico limpo, um ecossistema estável agora e sustentável em longo prazo - Cidade Saudável. De tal modo, iniciativas para uma Cidade Inteligente podem contribuir para a construção de uma Cidade Saudável e vice-versa, haja vista que as pessoas com altos níveis de saúde, educação, qualidade de vida etc. têm maior propensão a ser ativos nas mudanças das cidades.

A tabela 1 resume as principais semelhanças e diferenças entre as noções de Cidades Saudáveis e Cidades Inteligentes. 
Tabela 1: Aspectos principais de Cidades Saudáveis e de Cidades Inteligentes (2017).

Componentes
Década do surgimento
Origem do conceito
Principal característica
Objetivo
Foco
Abordagem
Infraestruturas e
serviços urbanos
Orientação
Ambiente/natureza
Papel do cidadão
Planejamento urbano
Políticas públicas
Proponente
Avaliação

Org. Elaborado pela autora (2017).

\section{Cidades Saudáveis \\ 1980 \\ OMS}

Promoção da saúde

Qualidade de vida, bem-estar sustentabilidade como quesito de desenvolvimento.

Pessoas

Botton up

Acessíveis

Longo prazo

Bem natural Ativo

Integrado

Intersetoriais

Iniciativa governamental
Cidades Inteligentes

1990

Iniciativa privada

Inovações e tecnologias

Qualidade de vida, bem-estar

sustentabilidade como quesito de

competividade.

Mercado

Top down e botton up

Conectados

Curto a longo prazo

Recurso natural

Ativo

Integrado

Intersetoriais

Iniciativa privada Indicadores

Os conceitos de Cidades Saudáveis e Cidades Inteligentes, são recentes, surgindo no final do século $\mathrm{XX}$, contexto marcado pela rápida expansão da população urbana e dos problemas dela decorrentes, juntamente com as mudanças econômicas e tecnológicas causadas pela globalização. O contexto, portanto representa, simultaneamente, uma conjuntura de crise socioeconômica e de possibilidades de mudança, haja vista as crescentes expectativas dos cidadãos e as novas oportunidades decorrentes dos crescentes avanços nas TIC's.

A noção de Cidades Saudáveis, mais antiga, tem sua origem no âmbito de uma iniciativa da OMS, portanto a partir da busca pela promoção da saúde por uma instituição pública de alcance internacional. A noção de Cidades Inteligentes, mais recente, surge a partir de empresas de tecnologia, portanto no âmbito da iniciativa privada, para referir-se à utilização das inovações e dos recursos tecnológicos na infraestrutura e serviços urbanos.

Quanto ao objetivo, independente do adjetivo utilizado para qualificar as cidades, em última instância o que se busca é o alcance da qualidade de 
vida, o bem-estar e a sustentabilidade. Contudo, uma análise crítica dos estudos, técnicos e científicos considerados para a redação do presente artigo, permite afirmar que no âmbito do conceito de Cidades Inteligentes, o alcance de tais condições atenderia a reprodução do capitalismo globalizado.

Para os estudiosos de vertentes marxistas, como Harvey (1989), as propostas de Cidades Inteligentes, de modo geral, não representam alternativa à cidade neoliberal, haja vista que parte de iniciativas empresariais se apropriam do rótulo "inteligente" como um dispositivo de marketing para a cidade e uma desculpa para a dominação dos modelos corporativos de empreendedorismo urbano, difundindo mundialmente um modelo de "Cidade Inteligente Corporativa". As cidades então passam a competir umas com as outras para atrair capital global e comercializar-se como cidades (HOLLANDS, 2008 apud HOLLANDS, 2014).

Como consequência pressupõe-se que nas Cidades Saudáveis o foco é a melhoria das condições ambientais, econômicas e de vida das pessoas, para a promoção da saúde. Já nas Cidades Inteligentes, este não é o foco principal, mas sim a promover a concentração de capital humano para atrair negócios e atividades que transformem as cidades em polos de competitividade global (MOSANNENZADEH; VETTORATO, 2014).

Ao considerar a forma de transformação das cidades, o conceito de Cidades Saudáveis tem como pressuposto o conhecimento do território pelas pessoas, de modo que possam agir sobre esse a seu favor. Logo, as estratégias devem partir da comunidade, que tem posição essencial na construção das políticas públicas junto aos governos. Desta forma, ressaltase que a abordagem bottom up tem papel central neste conceito. Por outro lado, ainda que se reconheça que esta abordagem também deve ocupar lugar fundamental no âmbito do conceito de Cidades Inteligentes, verifica-se que, muitas vezes, neste caso sobressai a abordagem top down, a partir da proposição da instalação de equipamentos tecnológicos por empresas, por vezes, sem ter em conta a participação dos cidadãos. 
Considerar a forma básica almejada para a infraestrutura e os serviços urbanos, pode auxiliar na compreensão destas abordagens predominantes. Nas Cidades Saudáveis, o acesso e, por conseguinte, a acessibilidade possui grande importância. Cabe aqui ressaltar que a acessibilidade compreende uma 'dimensão de acesso', portanto a qualidade de ser acessível, tanto na perspectiva da oferta como da adequação entre a distribuição geográfica dos serviços e dos cidadãos, considerando o tempo de viagem, as distâncias e os custos para chegar aos serviços (PENCHANSKY; THOMAS, 1981; DONABEDIAN, 2003).

Já nas Cidades Inteligentes a conexão entre os espaços e serviços é uma questão basilar. Sobretudo, a partir da Internet of Things (IoT), em português Internet das Coisas, cuja essência é conectar cada vez mais sensores, dispositivos e aparelhos que asseguram às pessoas a comunicação ea interação com outras pessoas e/ou objetos. Por exemplo, por meio de um conjunto de objetos, os padrões de mobilidade urbana são monitorados nas Cidades Inteligentes. Igualmente a iluminação, as lixeiras e os bueiros podem ser equipados com sensores que, por conseguinte, viabilizam sua conexão com outros dispositivos, tornando-os inteligentes.

$\mathrm{Na}$ perspectiva do alcance dos resultados, das condições de inteligente ou de saudável, as orientações para as ações também são distintas. No domínio das Cidades Inteligentes, especialmente considerando o ponto de vista da iniciativa privada, geralmente as intervenções visam retornos em horizontes de prazos mais curtos. Já nas Cidades Saudáveis fica explicita a ênfase no processo de melhoria contínua e nos horizontes de longo prazo.

Por fim, outro aspecto em que são notáveis as diferenças entre os conceitos de Cidade Saudável e Inteligente é em relação ao entendimento e tratamento dispensado ao ambiente, enquanto recurso da natureza. Neste caso, apesar de em ambos os conceitos estar expressa a preocupação com a preservação dos recursos naturais, de modo implícito pode-se notar que no primeiro predomina o entendimento da natureza como um bem natural 
essencial para a saúde e qualidade de vida, que é um direito de todos e não pode ser não monetarizado. No conceito de Cidade Inteligente, por sua vez, sobressai o entendimento da natureza como recurso natural, que atende as necessidades de reprodução da sociedade. Neste caso, o foco é a racionalidade, a eficiência e, por vezes, a valorização de determinadas áreas do espaço urbano que se destacam pelas amenidades ambientais, a preocupação com as relações sociais e com problemas socioambientais considerando a totalidade das cidades é secundária.

As cidades são entidades sócio-espaciais complexas (SOUZA, 2003) construídas e reconstruídas por diversos agentes sociais, dentre os quais destacam-se: os proprietários dos meios de produção, sobretudo os grandes industriais; os proprietários fundiários; os promotores imobiliários; o Estado e os grupos sociais excluídos (CORRÊEA, 1995). Destarte, independente do papel que ocupam na estrutura social, os cidadãos têm grande responsabilidade na (re)construção das cidades, considerando quaisquer que sejam os conceitos utilizados para designá-las. Neste sentido, ressalta-se que a mudança qualitativa das cidades é reflexo das condições sociais, em outras palavras, cidadãos saudáveis e/ou inteligentes (re)constroem Cidades Saudáveis e Inteligentes, respectivamente.

Nos projetos, como o de Fujisawa (Japão) tem-se enfatizado a busca pelo atendimento da necessidade do morador, bem como o valor da participação ativa dos cidadãos (SMART-CITIES, 2015). Afinal, estes devem ter a capacidade de ouvir o que a cidade diz, compreender como ela funciona e como deve ou não funcionar, com base no que querem ou não desejam.

O papel ativo do cidadão é valioso no contexto do processo de planejamento e gestão urbana, bem como na elaboração e implementação das políticas públicas locais, pois é a partir das menores escalas (cidade e/ou bairros), que o conhecimento e as ações individuais podem ter maior contribuição para o alcance daquele objetivo. Mas, esse processo também deve ser integrado, tanto no sentido vertical no âmbito das diversas políticas 
públicas locais, demandando, portanto políticas públicas intersetoriais, como na perspectiva horizontal, considerando as diversas escalas de materialização das políticas públicas.

Alcançar a condição de Cidade Saudável ou Inteligente pressupõe a busca por determinados objetivos essenciais como o alcance da qualidade de vida e do bem-estar, cuja evolução e resultado final devem ser assistidos a partir de indicadores de desempenho, que contribuem no processo de planejamento e gestão. Entretanto, um desafio é identificar adequadamente os indicadores que contemplem as diferentes dimensões, considerando tanto as abordagens de baixo para cima quanto as de cima para baixo, bem como que de fato sirvam para o monitoramento dos resultados e não como instrumento de marketing das cidades.

Neste sentido, tal como há grande variedade de iniciativas e abordagens são vários os indicadores utilizados tanto no âmbito das Cidades Saudáveis como das Cidades Inteligentes. Para as primeiras destacam-se a relevância dos indicadores de oferta e a acessibilidade aos estabelecimentos urbanos, especialmente aqueles que (in)diretamente viabilizam a promoção da saúde. Em relação a estas últimas, segundo Gartner (2017) (Líder mundial em pesquisa e consultoria em tecnologia da informação) a tendência é considerar indicadores de alteração climática, resiliência e sustentabilidade. Ao considerar ambas as tendências concorda-se com Giovannella; Dascalu e Scaccia (2014) quanto a pertinência de indicadores como tempo, que sinaliza melhoria na mobilidade, e o consumo de recursos, que refletem a conservação ambiental e eco-sustentabilidade, cujos indicadores podem ser monitorados tanto pelo uso de sensores índices quantitativos comode forma subjetiva pela percepção individual. 


\section{Considerações Finais}

O surgimento das noções de Cidades Saudáveis e de Cidades Inteligentes representam alternativas para resolver ou pelo menos mitigar grande parte dos problemas ambientais, sociais, políticos e econômicos das cidades no contexto do século XXI. Ao analisar os aspectos principais destas noções, como origem, definição, fundamentos e objetivos, identifica-se pontos de convergência e de divergência e as particularidades de cada noção.

Certamente a utilização das tecnologias, frutos da modernidade, para melhorar a funcionalidade dos serviços e infraestrutura urbanos, bem como para promover a integração dos diferentes sistemas de planejamento e a colaboração entre as várias partes interessadas são pressupostos essenciais para a criação de uma Cidade Inteligente. Porém, este pode ser um aspecto a ser mais valorizado no âmbito da noção de Cidade Saudável, haja vista que a articulação/conexão consiste em um atributo fundamental na/da sociedade contemporânea.

Por outro lado, um pressuposto básico da noção de Cidade Saudável é o papel ativo do cidadão, enquanto indivíduo que conhece as demandas do lugar onde vive e por isso pode ser determinante na mudança positiva deste e, por conseguinte, de suas condições de saúde, haja vista a intrínseca relação entre as condições do meio e as de saúde. Esta consiste em uma questão que deve ter destaque no âmbito das Cidades Inteligentes, que igualmente precisam considerar que os cidadãos são fundamentais na (re)construção das cidades, afinal a disseminação e utilização das tecnologias em uma cidade depende do capital social nela existente. Assim investimento na educação e valorização da democracia têm papel relevante na disseminação da utilização das tecnologias com vistas a melhoria das condições de vida.

Além disso, reforça-se que as iniciativas com vistas ao alcance de Cidade Inteligente podem contribuir com a construção de uma Cidade 
Saudável e vice-versa. Pois, como exposto cidades são sistemas complexos, onde as pessoas podem alcançar altos níveis de saúde, educação, qualidade de vida etc. através da utilização de conhecimento e recursos tecnológicos. Do mesmo modo, melhores condições de vida podem viabilizar o desenvolvimento e a adesão às inovações cientificas e tecnológicas, o que pode promover melhores condições de saúde, em um processo cíclico.

Enfim, ratifica-se ainda que a materialização de uma condição, adjetivada com inteligente ou mesmo saudável e/ou sustentável, depende do esforço de vários setores, instituições e de políticas intersetoriais (MOSANNENZADEH; VETTORATO, 2014). Portanto, em síntese, uma cidade ou região, saudável e/ou inteligente deve ser capaz de adquirir e aplicar conhecimentos para enfrentar os múltiplos desafios futuros.

\section{Referências}

ANTHOPOUlOS, L. G. Understanding the Smart City Domain: A Literature Review. In: RODRÍGUEZ-BOLÍVAR, M. P. (Org.).Transforming City Governments for Successful Smart Cities. Granada: Springer International Publishing, 2015. https://doi.org/10.1007/978-3-319-03167-5_2

ANTHOPOULOS, L. G. Smart Cities and Their Roles in City Competition: A Classification. International Journal of Electronic Government Research (IJEGR), v.10, n.1, 2014. https://doi.org/10.4018/ijegr.2014010105

ANTHOPOULOS, L. G.; FITSILIS, P. Smart Cities and their roles in city competition: A classification. International Journal of Electronic Government Research (IJEGR), v.10, n.1, p. 63-77, 2014BARTON, H., et al. Healthy urban planning in European cities.

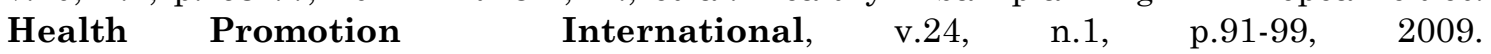
https://doi.org/10.4018/ijegr.2014010105

BUSS, P. Promoção da saúde e qualidade de vida. Ciência \& Saúde Coletiva, v.5, n.1, p.163-177, 2000. https://doi.org/10.1590/S1413-81232000000100014

BUSS, P. M.; CARVALHO, A. I. de. Desenvolvimento da promoção da saúde no Brasil nos últimos vinte anos (1988-2008). Ciência \& Saúde Coletiva, v.14, n.6, p.2305-2316, 2009. https://doi.org/10.1590/S1413-81232009000600039

COMISSÃO EUROPEIA. Smart Cities and Communities: The European Innovation Partnership on Smart Cities and Communities. Bruxelas, 2015.

CORRÊA, R. L. O Espaço Urbano. São Paulo, Editora Ática, 1995.

DAMERI, R. P. Searching for Smart City definition: a comprehensive proposal. International Journal of Computers \& Technology, v.11, n.5, p. 2544-2551, 2013. https://doi.org/10.24297/ijct.v11i5.1142

DONABEDIAN, A. An introduction to quality assurance in health care. New York: Oxford University, 2003.

DUHL, L. J. The healthy city: its function and its future. Health Promotion, v.1, p.55-60, 1986EXEMPLOS de Cidades Inteligentes no Brasil que vão te dar orgulho. Automatic 
House, 2019. Disponível em: $<$ https://www.automatichouse.com.br/imprensa/exemplos-decidades-inteligentes-no-brasil-que-vao-te-dar-orgulho/20190328-134649-v508> Acesso em: 15 jun. 2019.

FROST \& SULLIVAN'S. Strategic Opportunity Analysis of the Global Smart City Market. 2013. Disponível em: <http://www.frost.com/sublib/>. Acesso em: 15 mai. 2015.

GARTNER. Hype Cycle for Smart City Technologies and Solutions, 2017. Página inicial. Disponível em: <https://www.gartner.com/en/>. Acesso em: 20 de jun. de 2018.

GIOVANNELLA, C.; DASCALU, M.; SCACCIA, F. Smart City Analytics: state of the art and future Perspectives. Interaction Design and Architecture (s) Journal, n.20, p.7287, 2014.

HALL, C. DAVIES, J. K., SHERRIFF, N. Health in the Urban Environment: A Qualitative Review of the Brighton and Hove WHO Healthy City Program. Journal Urban Health. v.87, n.1, p.8-28, 2010. https://doi.org/10.1007/s11524-009-9387-y

HANCOCK, T. The Evolution, Impact and Significant of the Healthy Cities/Healthy, Communities Movement', Journal of Public Health Policy, v.14, n.1, p.5-18, 1993. https://doi.org/10.2307/3342823

HERITAGE, Z.; GREEN, G. European National Healthy City Networks: the Impact of an Elite Epistemic Community. Journal of Urban Health: Bulletin of the New York Academy of Medicine, v. 90, Suppl. 1. p.154-166, 2013. https://doi.org/10.1007/s11524-012$\underline{9777-4}$

HOLLANDS, R. G. Critical interventions into the corporate Smart City. Cambridge Journal Regions Economy Society, v.8, n.1, p. 61-77, 2014. https://doi.org/10.1093/cjres/rsu011

IBM- International Business Machines. Cidades Inteligentes e seus impactos econômicos, 2011. Disponível em: <https://www.ibm.com>. Acesso em: 15 jan. 2017.

KOOLHAS, R. My thoughts on the Smart City. Edited transcript of a talk given at the High Level Group meeting on Smart Cities, Brussels, 24 September 2014. Disponível em $<$ http://ec.europa.eu/archives $>$. Acesso em: 15 jan. 2017.

LEFEBVRE, H. A Revolução Urbana. Tradução de Sérgio Martins. Belo Horizonte: UFMG, 1999.

LIMA, M. Uberlândia ganha protótipo de Cidade Inteligente. Revista Inova. Disponível em: <http://www.inova.jor.br/2016/12/14/algar-uberlandia-cidade-inteligente>. Acesso em: 15 jan. 2017.

MYFANWY; T. Working paper for the lancet. Commission on healthy cities. Department of Geography, University College London, 2010.

MOSANNENZADEH, F.; VETTORATO, D. Defining Smart City: a conceptual framework based on keyword analysis. TeMA Journal of Land Use Mobility and Environment. INPUT 2014 Eighth International Conference INPUT - Naples, 4-6 June 2014.

NAM, T.; PARDO; T. A. Smart City as urban innovation: focusing on management, policy, and context. In: ICEGOV ' 11 Proceedings of the 5th International Conference on Theory and Practice of Electronic Governance. 5, Estonia. Anais.... Tallinn: 2011, p.185-194. https://doi.org/10.1145/2072069.2072100

OPAS - Organización Panamericana De La Salud. Desarrollo de los sistemas locales de la salud. Washington, D.C., 1996

PANASONIC. Panasonic inaugura a sua Cidade Inteligente e Sustentável em Fujisawa. 27-11-2014. Disponível em: <https://www.panasonic.com/pt>. Acesso em: 15 jan. 2017.

PENCHANSKY, D. B. A.; THOMAS, J. W. The concept of access - definition and relationship to consumer satisfaction. Med Care, Hagerstown, v. 19, p. 127-140, 1981. https://doi.org/10.1097/00005650-198102000-00001

REDE BRASILEIRA DE CIDADES INTELIGENTES E HUMANAS. Institucional. 2017. Disponível em: <http://redebrasileira.org/home>. Acesso em: 15 jan. 2017.

RIGHETTI, S. Condições ambientais e bem estar social são fatores que influenciam saúde da população. Cienc. Cult. [online]. v.56, n.2, p. 06-07, 2004. 
SASSEN, S. Las ciudades van a ser más importantes que los estados. Entrevista a ABC. 15 mai. 2013. Disponível em: <http://www.abc.es/20120612>. Acesso em: 14 jun. 2016. SHELTON, T.; ZOOK, M.; WIIG, A. The 'actually existing Smart City'. Cambridge Journal of Regions, Economy and Society, v. 8, n. 1, p.13-25, 2015. https://doi.org/10.1093/cjres/rsu026

SOUZA, M. L. ABC do desenvolvimento urbano. Rio de Janeiro: Bertrand Brasil, 2003. WHO -World Health Organization. City planning for health and sustainable development. European Sustainable Development and Health Series, 2. 1997. 111p. Regional Office for Europe. Implementing a Health 2020 vision: governance for health in the 21st century. Making it haplicativoen, 2013b.

. European Healthy Cities Network. 2014. 